Published in final edited form as:

Dig Dis Sci. 2012 August ; 57(8): 2213-2221. doi:10.1007/s10620-012-2171-y.

\title{
The Association of Genetic Variants with Hepatic Steatosis in Patients with Genotype 1 Chronic Hepatitis C Infection
}

\author{
Paul J. Clark \\ Duke Clinical Research Institute, Duke University, PO Box 17969, Durham, NC 27715, USA \\ Kirby Institute for Infection and Immunity in Society, University of New South Wales, Kensington, \\ Australia
}

\author{
Alexander J. Thompson \\ Duke Clinical Research Institute, Duke University, PO Box 17969, Durham, NC 27715, USA \\ Qianqian Zhu \\ Center for Human Genome Variation, Duke University, Durham, NC, USA \\ David M. Vock \\ Duke Clinical Research Institute, Duke University, PO Box 17969, Durham, NC 27715, USA \\ Mingfu Zhu \\ Center for Human Genome Variation, Duke University, Durham, NC, USA
}

Keyur Patel

Duke Clinical Research Institute, Duke University, PO Box 17969, Durham, NC 27715, USA

\author{
Stephen A. Harrison \\ Brooke Army Medical Center, Fort Sam Houston, TX, USA
}

\section{Susanna Naggie}

Duke Clinical Research Institute, Duke University, PO Box 17969, Durham, NC 27715, USA

\section{Dongliang Ge}

Center for Human Genome Variation, Duke University, Durham, NC, USA

\section{Hans L. Tillmann}

Duke Clinical Research Institute, Duke University, PO Box 17969, Durham, NC 27715, USA

Thomas J. Urban

Center for Human Genome Variation, Duke University, Durham, NC, USA

Kevin Shianna

Center for Human Genome Variation, Duke University, Durham, NC, USA

\footnotetext{
(C) Springer Science+Business Media, LLC 2012

drpjclark@gmail.com.

muir0002@mc.duke.edu
}

Electronic supplementary material The online version of this article (doi:10.1007/s10620-012-2171-y) contains supplementary material, which is available to authorized users.

Conflict of interest The authors disclose the following: Drs. Thompson, Harrison, Sulkowski, Afdhal, McHutchison and Muir report having received research and grant support from Schering-Plough (now Merck); Drs Afdhal, Harrison, McHutchison, Goldstein and Muir have received consulting fees or acted in an advisory capacity for Schering-Plough or Merck. Dr Harrison has received grant and research support from Genentech, Merck, and Rottapharm, worked in an advisory capacity for Three Rivers Pharmaceuticals and Amylin and for speaker's bureau for Bristol Myers Squibb and Merck. Drs Albrecht and Pedicone are employees of Schering-Plough (now Merck \& Co.) and are stockholders in this entity. Dr McHutchison is now employed by Gilead Sciences. Dr Noviello is a former employee of Schering-Plough and is now a consultant to Merck \& Co. Drs. Thompson, Ge, Urban, McHutchison and Goldstein are co-inventors of a patent application based on the IL28B finding. 
Jacques Fellay

Center for Human Genome Variation, Duke University, Durham, NC, USA

Zachary Goodman

Armed Forces Institute of Pathology, Washington, DC, USA

Stephanie Noviello

Schering-Plough Corporation (now Merck \& Co., Inc.), Whitehouse Station, NJ, USA

Lisa D. Pedicone

Schering-Plough Corporation (now Merck \& Co., Inc.), Whitehouse Station, NJ, USA

Nezam Afdhal

Beth Israel Deaconess Medical Center, Boston, MA, USA

Mark Sulkowski

Johns Hopkins University School of Medicine, Baltimore, MD, USA

Janice K. Albrecht

Schering-Plough Corporation (now Merck \& Co., Inc.), Whitehouse Station, NJ, USA

David B. Goldstein

Center for Human Genome Variation, Duke University, Durham, NC, USA

John G. McHutchison

Duke Clinical Research Institute, Duke University, PO Box 17969, Durham, NC 27715, USA

Andrew J. Muir

Duke Clinical Research Institute, Duke University, PO Box 17969, Durham, NC 27715, USA

\section{Abstract}

Background-Single-nucleotide polymorphisms (SNPs) in the IL28B and PNPLA3 gene regions have been associated with hepatic steatosis in genotype 1 (G1) chronic HCV infection but their clinical impacts remain to be determined.

Aim-We sought to validate these associations and to explore their impact on treatment response to peginterferon and ribavirin therapy.

Methods-A total of $972 \mathrm{G} 1 \mathrm{HCV}$-infected Caucasian patients were genotyped for the SNPs rs 12979860 (IL28B) and rs2896019 (PNPLA3). Multivariable analysis tested IL28B and PNPLA3 for association with the presence of any steatosis $(>0 \%)$; clinically significant steatosis $(>5 \%)$; steatosis severity (grade 0-3/4); and the interacting associations of the SNPs and hepatic steatosis to sustained viral response (SVR).

Results-IL28B and PNPLA3 polymorphisms were associated with the presence of any steatosis (rs12979860, $\left.p=1.87 \times 10^{-7} ; \mathrm{rs} 2896019, p=7.56 \times 10^{-4}\right)$; clinically significant steatosis $\left(\mathrm{rs} 12979860, p=1.82 \times 10^{-3} ; \mathrm{rs} 2896019, p=1.27 \times 10^{-4}\right)$; and steatosis severity (rs12979860, $p$ $=2.05 \times 10^{-8} ;$ rs $2896019, p=2.62 \times 10^{-6}$ ). Obesity, hypertriglyceridemia, hyperglycemia, liver fibrosis, and liver inflammation were all independently associated with worse steatosis. Hepatic steatosis was associated with lower SVR, and this effect was attenuated by IL28B. PNPLA3 had no independent association with SVR.

Conclusions - IL $28 B$ and PNPLA3 are associated with hepatic steatosis prevalence and severity in Caucasians with $\mathrm{G} 1 \mathrm{HCV}$, suggesting differing potential genetic risk pathways to steatosis. IL28B attenuates the association between steatosis and SVR. Remediable metabolic risk factors remain important, independently of these polymorphisms, and remain key therapeutic goals to achieve better outcomes for patients with HCV-associated hepatic steatosis. 


\section{Keywords}

Polymorphism, single-nucleotide, SNP; IL28B protein, human; PNPLA3 protein, human;

Adiponutrin, human; Fatty liver; Abdominal obesity metabolic syndrome

\section{Introduction}

Hepatic steatosis is frequently observed in the setting of chronic hepatitis C virus (HCV) infection and has been associated with poor treatment response (SVR, sustained viral response) and more advanced hepatic fibrosis [1-3]. In genotype $3 \mathrm{HCV}$ infection, viral factors are implicated in the development of steatosis, while for genotype 1 (G1) HCV, host metabolic risk factors such as obesity and insulin resistance afford increased risk $[4,5]$.

The increasing burden of chronic HCV infection, in combination with an epidemic of obesity in the West, suggests that hepatic steatosis will have an increasing contribution to liver disease [6-10]. Better understanding of the interactions between host genetic and metabolic risk factors for HCV-related steatosis is thus important.

Recently, an association between $I L 28 B$ polymorphism and hepatic steatosis in Caucasian patients with mixed HCV genotype infection (324 patients with G1 HCV) was recognized [11]. The relatively small proportion of G1 HCV patients limited exploration of the interrelationships between these SNPs, hepatic steatosis, and treatment outcomes. Our group also reported the association with $I L 28 B$ and hepatic steatosis in a combined clinical and trial cohort, but again only a limited number of patients had SVR data $(n=164)$ [12]. A nonsynonymous polymorphism in the patatin-like phospholipase domain-containing 3 gene (PNPLA3 rs738409, C > G encoding I148 M,) has been associated with hepatic fat content in non-alcoholic fatty liver disease (NAFLD) $[13,14]$ and histological features of NAFLD severity [15], though its functional mechanism remains unclear. European candidate gene studies have confirmed the association with PNPLA3 and hepatic steatosis in chronic HCV infection, further correlating the risk allele with hepatic fibrosis, though with conflicting results regarding the association between PNPLA3 and SVR $[11,16,17]$. We sought to validate the associations of $I L 28 B$ and PNPLA3 on G1 HCV-associated hepatic steatosis, and to assess their impact on SVR, in a large, well-characterized clinical trial cohort.

\section{Methods}

\section{Patients}

The IDEAL trial was a multi-center, randomized control trial that demonstrated similar efficacy and adverse events of differing regimens of peginterferon-alfa and ribavirin (PEG/ RBV) in 3,070 North American patients with chronic G1 HCV (ClinicalTrials.gov number, NCT00081770) [18]. A pharmacogenomics sub-cohort from the trial consented to DNA testing and has been previously analyzed for genome-wide associations with treatment response [19] and ribavirin-induced hemolytic anemia [20]. To explore and validate previous European Caucasian genetic associations studies, the analysis was limited to Caucasians $(n=972)$. Clinical characteristics of the study population are presented in Table 1.

Steatosis was blindly evaluated by a single expert histopathologist (ZG) and graded on the percentage of hepatocytes with steatosis [grade $0(0 \%$ hepatocytes); $1(>0-5 \%) ; 2(>5-32$ $\%) ; 3$ (>32-66 \% hepatocytes affected); or 4 (>66\% hepatocytes affected)]. For inclusion in this study, patients required a minimum of six portal tracts in the liver biopsy. The mean biopsy length was $21 \mathrm{~mm}$, with $59 \%$ having liver biopsies more than $15 \mathrm{~mm}$ in length. The 
mean body mass index (BMI) was $27.8 \mathrm{~kg} / \mathrm{m}^{2}$, with over a quarter of patients classified as obese (BMI > $30 \mathrm{~kg} / \mathrm{m}^{2}$ ). In keeping with what one might expect clinically in a G1 HCV cohort, hepatic steatosis was typically not severe $(15 \%>$ grade 1$)$.

As per the IDEAL trial protocol, a history of alcohol consumption of more than $80 \mathrm{~g} / \mathrm{day}$ within 2 years of enrollment, or histological evidence of significant steato-hepatitis were exclusion criteria [18]. Patients on statin therapy at any time during the study period were excluded from the genetic analysis to avoid potential confounding $(n=46)$.

\section{Genetic Analysis}

Patients were genotyped using the Illumina Human610- quad BeadChip. Ethnicity was genetically inferred, and principal components analysis was conducted using a modified Eigenstrat method to correct for population sub-stratification [21]. Full genotyping procedures and quality-control measures undertaken have been previously described in the original study from which this dataset was extracted [19]. Here we validate genetic associations of IL28B (rs12979860) and PNPLA3 (using proxy SNP rs2896019) with HCVassociated hepatic steatosis in Caucasian patients, using conventional statistical significance criteria $(p<0.05)$.

rs12979860 variant, upstream from the $I L 28 B$ gene, is a biallelic variant where CC genotype is associated with better treatment response and genotypes with the T allele (CT and TT variants/genotypes) associated with relatively poorer treatment response. Unfortunately, the PNPLA3 variant rs738409 is not genotyped directly on the Illumina Human610- quad BeadChip. Using open access software (SNAP 2.2, Broad Institute, Harvard University, MA, USA) [22], we searched the 1,000 Genomes Reference database [23] for an alternative, proxy SNP present on the GWAS chip that was correlated (in linkage disequilibrium, LD) with the PNPLA3 variant rs738409. The SNP in strongest LD with rs738409 identified on the chip was rs $2896019\left(R^{2}=0.688, \mathrm{D}^{\prime}=0.94\right)$.

Genome analysis was conducted with PLINK software and additional SVR analyses was conducted with STATA (StataCorp, College Station, TX, USA) and SAS 9.2 (SAS Institute, Cary, NC, USA) software [24].

\section{Phenotype Definition of Hepatic Steatosis}

Hepatic steatosis was modeled in three ways. Firstly, to assess whether genetic variants were protective for steatosis, we assessed the total absence of hepatic steatosis (binary variable in logistic regression model, $0 \%$ or greater than $0 \%$ hepatocytes with steatosis). Next, we assessed "clinically significant" steatosis using conventionally accepted definitions (binary variable/logistic model, $0-5 \%$ vs. greater than $5 \%$ steatosis). Finally, we assessed severity or grade of hepatic steatosis [ordinal logistic regression model, with grades 3 and 4 combined to satisfy proportional hazards assumptions (Supplementary Table 1)].

Covariates adjusted for in the models included age, gender, body mass index $\left(\mathrm{kg} / \mathrm{m}^{2}\right)$, baseline HCV viral $\operatorname{load}_{(}\left(\log _{10} \mathrm{IU} / \mathrm{ml}\right)$, fibrosis (binary variable as METAVIR stage F0-2 vs. F3-4), inflammation (METAVIR grade A0-1 vs. A2-3), alanine transaminase (ALT) values, baseline fasting blood glucose levels and genetically defined ancestry sub-population sets (or Eigenstrat vectors). Plasma HCV RNA concentrations were measured using the COBAS Taqman assay with a lower limit of quantitation of $27 \mathrm{IU} / \mathrm{ml}$ (Roche Diagnostics, Indianapolis, IN, USA). 


\section{Results}

\section{IL28B and PNPLA3 Variants Are Associated with Genotype-1 HCV-Induced Hepatic Steatosis Prevalence and Steatosis Severity}

Genotype frequencies for IL28B rs12979860 were: TT: 117 (12.1\%); TC: 490 (50.8 \%); CC: $357(37.0 \%)$. Patients with the T allele had significantly more steatosis across all grades of steatosis (Fig. 1). The presence of any steatosis $(>0 \%)$ was significantly associated with the poor response T allele (OR: $1.88, p=1.87 \times 10^{-7}$, Table 1$)$. Clinically significant hepatic steatosis ( $>5 \%$ steatosis $)\left(p=1.82 \times 10^{-3}\right)$ and hepatic steatosis severity were also both associated with the T allele (OR 1.80, (95\%CI 1.47-2.21), $p=2.05 \times 10^{-8}$, Table 2).

Genotype frequency for the PNPLA3 proxy SNP rs2896019 (where the $\mathrm{G}$ allele is associated with steatosis risk) were: GG: 26 (2.7 \%), GT: 264 (27.2\%) and TT: 682 (70.2 $\%$ ). Univariate analysis demonstrated patients with the $\mathrm{G}$ allele had proportionally more steatosis for each grade, and proportionally less were free of steatosis (grade 0, 33.1 vs. 42.7 $\% \mathrm{G}$ allele vs. TT genotype, respectively, Fig. 2). On Logistic regression multivariable analysis (MVA), PNPLA3 was independently associated with the presence of any steatosis (OR (G allele): 1.62, (95\% CI 1.22-2.14), $p=7.56 \times 10^{-4}$, Table 3); the presence of clinically significant hepatic steatosis $\left(p=1.27 \times 10^{-4}\right)$; and more severe steatosis $(\mathrm{OR} 1.78$ (95\% CI 1.40-2.27) $p=2.62 \times 10^{-6}$, Table 3 ).

Finally, we sought to assess how the additional genetic information improved the model's explanation of variability in steatosis severity (as measured by pseudo $\mathrm{R}^{2}$ ). Inclusion of either or both PNPLA3 and IL28B SNPs provided very modest improvement in model performance as indicated by relative change in the pseudo $\mathrm{R}^{2}$ (Table 4 ).

\section{Host Metabolic Risk Factors Are the Major Determinants of Hepatic Steatosis}

BMI had the most statistically significant association with steatosis severity (IL28B model: OR 1.11, (95\% CI 1.07-1.14), $p=4.78 \times 10^{-11}$; PNPLA3 model: OR 1.11, (95\% CI 1.07, $1.15), 1.41 \times 10^{-11}$, Tables 2,3 ). Other clinical variables had significant association with the respective steatosis severity models, with differing effect sizes, including age, histological evidence of advanced hepatic fibrosis and inflammation, elevated ALT, elevated baseline fasting glucose and baseline triglycerides.

We explored whether these genetic associations may be working indirectly through other steatosis risk factors, or interacting with each other. For its association with steatosis severity, IL28B showed interaction with alanine aminotransferase (ALT) $(p=0.003)$; triglyceride (TG) levels $(p<0.0001)$; fasting glucose levels $(p=0.045)$ and fibrosis $(p<0.0001)$. However, these factors differed minimally when we compared univariate analysis between patients with the good response to poor response variants, with minor differences only in ALT levels (2.6 vs. 2.0 times the upper limit of normal, respectively, $p<0.0001$ ) and fasting blood glucose levels, (mean glucose level 5.14 vs. $5.31 \mathrm{mmol} / \mathrm{l}$, respectively, $p=0.0066$ ). We observed no interaction between the effect of PNPLA3 and IL28B for steatosis. On sub-analysis limited to patients with the PNPLA3 risk allele (rs2896019 TG or GG), the $p$ value for association between rs 12979860 and steatosis is 0.00013345 based on logistic regression, while for patients without the risk genotype (rs2896019 TT genotype), for the association between rs12979860 and steatosis the $p$ value was 0.00103807 .

PNPLA3s effect on steatosis was not influenced by fibrosis, BMI, ALT, TG or glucose levels, in its association with steatosis severity (interaction $p$ values all non-significant), thus offering little clue to pathogenic pathways linking PNPLA3 to steatosis. The PNPLA3 effect 
on steatosis was influenced by age ( $p=0.017)$, but age distributions did not differ when compared between genotypes.

PNPLA3 variant's association with hepatic fibrosis was dependent on the genetic model used (rs2896019 association with>F2 fibrosis, recessive model GG vs. non-GG: $p=0.695$; dominant model GG and TG vs. TT: $p=0.040$; and, additive model GG vs. GT vs. TT: $p=$ $0.089)$.

\section{IL28B Attenuates the Impact of Hepatic Steatosis on SVR but PNPLA3 Has No Significant Association with SVR Directly or Indirectly Through Steatosis}

The absence of clinically significant steatosis ( $5 \%$ hepatocytes) was associated with improved SVR in a multivariable model that did not include IL28B or PNPLA3 genotype (OR 2.20 (95\% CI 1.39-3.47) $p=0.001$, Supplementary Table 2). After the inclusion of $I L 28 B$ as a covariate into the model, the effect and significance of hepatic steatosis on treatment response was attenuated, however, steatosis remained an independently significant predictor (OR 1.73 (95\%CI 1.05-2.83) $p=0.031$, Supplementary Table 3).

No significant independent association between PNPLA3 and SVR was observed in a multivariable analysis $(p=0.294)$ and the inclusion of PNPLA3 in the model did not alter the strength or significance of the association between steatosis and SVR (Supplementary Table 4). We ran this same model for the presence of any steatosis (>0\%) and there was similarly no significant relationship. Thus, on MVA we were unable to identify a direct association between PNPLA3 and SVR, nor find any evidence of PNPLA3 indirectly altering the effect of steatosis on SVR.

Finally, we assessed whether PNPLA3 was significant when analysis was limited to those patients with advanced fibrosis ( $\triangle$ METAVIR F3). In this limited subgroup (limited further by available response data, $n=85$ ), we found no association between PNPLA3 and SVR ( $p$ $=0.288$ ). Interestingly, for these patients with advanced fibrosis, the only significant predictors of SVR were total cholesterol (OR 1.82; $95 \%$ CI: $0.67-4.75 ; p=0.033$ ) and fasting blood sugar (OR 2.40 (per unit decrease mmol/1); $95 \%$ CI: 1.09-5.29; $p=0.029$ ), but not HCV RNA burden $(p=0.408)$.

\section{Discussion}

We have shown that the prevalence and severity of hepatic steatosis in Caucasian patients with G1 HCV is associated with IL28B and PNPLA3 genotypes. Our findings are consistent with previous candidate-gene association studies [11, 12, 16, 17]. Our results also suggest that even allowing for these significant genetic associations, metabolic and clinical risk factors continue to be significant for $\mathrm{HCV}$-induced hepatic steatosis. We have shown that hepatic steatosis remains independently associated with SVR even after consideration of host genetic variants, though importantly, the association between steatosis and SVR is attenuated by $I L 28 B$. We found no association between the PNPLA3-proxy variant and SVR or fibrosis.

For clinicians, these results may help to place in context the clinical impacts of host genetic variation on hepatic steatosis. This data supports an important yet relatively modest role for host genetic variation explaining the variability of HCV-associated steatosis. Our study reiterates the clinical link between less steatosis and improved treatment responses, and may support attempts to motivate patients with lifestyle modification such as weight loss [25, 26]. Persistent fatty liver disease can remain a problem for patients with G1 HCV even with successful viral clearance [2,3]. Thus, despite higher SVR rates anticipated in the era of $\mathrm{HCV}$ direct acting antiviral agents, hepatic steatosis may remain a clinical problem even for 
patients cured of HCV. For patients, this may mean a "transition" from one primary liver disease (HCV) to another (NAFLD), further underscoring the importance for clinicians to seize opportunities to optimize metabolic risk factors. Host IL28B/PNPLA3 genotype may help to identify those patients at increased risk of steatosis, and consequently those who may benefit most from lifestyle intervention.

It is important to note the differences in this analysis compared to previously reported candidate-gene associations with hepatic steatosis. A major caveat to our results was the use of a proxy-SNP for the PNPLA3 variant rs738409. Despite this, we demonstrated significant associations between PNPLA3 and all steatosis phenotypes, but not for SVR nor convincingly so for fibrosis. While acknowledging the possibility of false-negative associations for fibrosis and SVR due to the use of a proxy SNP, our results show some consistency with others' findings. Trepo et al. [17] demonstrated an association between PNPLA3 and hepatic steatosis and fibrosis in multivariate models with dependence on the genetic model and no significant relationship was found between PNPLA3 and SVR. In contrast, we found a significant relationship with the primary phenotype of steatosis in our proxy SNP irrespective of the model used, suggesting the SNP was a valid candidate to study for association. Trepo et al. found no association between IL28B (rs12979860) and hepatic steatosis in contrast to our highly significant association across a variety of phenotypes, further suggesting that cohort differences may be important in differing genetic association results rather than sub-optimal linkage disequilibrium of the candidate SNP.

Our study had the advantages of a large sample size, with uniform, protocol-based assessment. In particular, liver biopsy assessment was by a single, expert histopathologist, thus reducing inter-observer variability. Our cohort had proportionally less patients with advanced hepatic fibrosis than these European studies (11.6\% of this cohort with $>\mathrm{F} 2$ METAVIR stage compared to $26-40 \%$ ), $[11,16,17]$ and thus potentially underpowered to find associations with fibrosis, or in subgroup analysis of SVR in patients with advanced fibrosis. PNPLA3 has been associated with alcohol-related liver injury and cirrhosis, and alcohol is an important co-factor in liver injury and fibrosis in chronic HCV infection [2729]. PNPLA3 clinical associations with steatosis and fibrosis in chronic HCV are modulated, though not dependent on alcohol use [30,31]. Significant alcohol intake was an exclusion criterion for this clinical trial cohort study, while for instance in the Swiss study a third of patients reported consuming more than $20 \mathrm{~g}$ of alcohol per day [11]. Importantly, we were not able to quantify alcohol exposure except as less than $80 \mathrm{~g}$ per day, yet doses less than this may contribute significantly to steatosis, and were not captured by our study. While we were able to consider for fasting glucose, the absence of insulin resistance data was a major limitation, and necessary to qualify the analyses of both steatosis and SVR. Finally, over a quarter of our cohort were obese (BMI>30 mg/ $\mathrm{kg}^{2}$ ) with a mean BMI of $27.8 \mathrm{mg} / \mathrm{kg}^{2}$, higher than comparative European cohort studies where median BMI varied from 23.7 to $25.9 \mathrm{mg} / \mathrm{kg}^{2}[11,16,17]$. Thus, a range of cohort factors including rates of hepatic fibrosis, contributing alcohol use, and obesity may provide differing environmental contributions to steatosis, and thus influence the relative contribution of host genetics. While reflecting the heterogeneity of genetic and environmental contributions to steatosis and fibrosis, it also underscores the importance of validating host genetic-clinical associations in variety of clinical contexts and populations.

The functional underpinning of the IL28B and PNPLA3 associations with HCV-induced steatosis is not clear. A weak correlation has previously been found between interferonstimulated gene expression and hepatic steatosis [32], and higher levels of ISG expression are correlated with the unfavorable $I L 28 B$ genotypes [33-35]. This association may therefore represent a non-specific association between intrahepatic inflammation/ISG induction, and this might be reflected by the significant interaction between ALT and IL28B 
for steatosis. PNPLA3 mRNA expression has been found to be decreased in patients with advanced fibrosis or cirrhosis from alcohol and non-alcoholic fatty liver disease but intriguingly, PNPLA3 gene expression did not differ by PNPLA3 genotype, nor does PNPLA3 genotype differentiate the expression patterns of other candidate-gene pathways involved in steatosis, adipogenesis, or fibrogenesis. While it is possible to speculate on inflammatory pathways as a possible link between IL28B and hepatic steatosis, hypotheses on potential pathogenic links between PNPLA3 and steatosis are less clear. Further exploration of these functional pathways and genotype-based differential gene expression patterns may help understand their pathogenesis in $\mathrm{HCV}$-associated hepatic steatosis.

Our observations help to clarify the role of genetic variants in G1 HCV-associated hepatic steatosis and reflect its complex, multifactorial nature. We confirm significant genetic associations between IL28B and PNPLA3 and hepatic steatosis. We show that beneficial $I L 28 B$ genotype attenuates the negative effect of hepatic steatosis with SVR, but found no equivalent relationship for PNPLA3. We found no significant interaction between these SNPs in their effect on steatosis in contrast to recent reports, where IL28B association with steatosis was apparent only in patients without the G risk allele for PNPLA3 (rs738409) [36]. In these multivariable models, remediable risk factors such as BMI and fasting plasma glucose all remain independently significant, identifying important therapeutic opportunities to help patients modify risks. For patients with G1 HCV, improving hepatic steatosis remains an important clinical goal, irrespective of host genotype. At the population level, development of effective, lifestyle interventions will be critical to improve hepatic steatosis, achieve better HCV treatment responses and importantly, better liver health after HCV cure.

\section{Acknowledgments}

The authors would like to thank the patients in the IDEAL trial as well as the principal investigators, study coordinators, and nurses involved. The authors thank Megan Koehler, DCRI for her statistical review. The study was funded by Schering-Plough Research Institute, Kenilworth, NJ (now Merck and Co.). Drs. Clark and Thompson received funding support from the Duke Clinical Research Institute, the Richard Boebel Family Fund, the National Health and Medical Research Council of Australia (PJC: APP1017139), the Gastroenterological Society of Australia. Dr Thompson received funding from the Royal Australian College of Physicians. Dr. Clark received funding from the National Centre in HIV Epidemiology and Clinical Research (now The Kirby Institute for Infection and Immunity in Society), University of New South Wales, Australia, and the AASLD/LIFER Clinical and Translational Research Fellowship in Liver Diseases Award.

This study is conducted for ideal investigators.

\section{Abbreviations}

$\begin{array}{ll}\text { HCV } & \text { Hepatitis C virus } \\ \text { SNP } & \text { Single-nucleotide polymorphism } \\ \text { IL28B } & \text { Interleukin 28B } \\ \text { PNPLA3 } & \text { Patatin-like phospholipase domain-containing 3 } \\ \text { G1 } & \text { Genotype 1 } \\ \text { MVA } & \text { Multivariable analysis } \\ \text { SVR } & \text { Sustained viral response } \\ \text { NAFLD } & \text { Non-alcoholic fatty liver disease }\end{array}$




\section{References}

1. Hourigan LF, Macdonald GA, Purdie D, et al. Fibrosis in chronic hepatitis C correlates significantly with body mass index and steatosis. Hepatology. 1999; 29:1215-1219. [PubMed: 10094967]

2. Poynard T, Ratziu V, McHutchison J, et al. Effect of treatment with peginterferon or interferon alfa-2b and ribavirin on steatosis in patients infected with hepatitis C. Hepatology. 2003; 38:75-85. [PubMed: 12829989]

3. Patton HM, Patel K, Behling C, et al. The impact of steatosis on disease progression and early and sustained treatment response in chronic hepatitis C patients. J Hepatol. 2004; 40:484-490. [PubMed: 15123364]

4. Hui JM, Kench J, Farrell GC, et al. Genotype-specific mechanisms for hepatic steatosis in chronic hepatitis C infection. J Gastroenterol Hepatol. 2002; 17:873-881. [PubMed: 12164963]

5. Fartoux L, Poujol-Robert A, Guéchot J, Wendum D, Poupon R, Serfaty L. Insulin resistance is a cause of steatosis and fibrosis progression in chronic hepatitis C. Gut. 2005; 54:1003-1008. [PubMed: 15951550]

6. Hu K-Q, Kyulo NL, Esrailian E, et al. Overweight and obesity, hepatic steatosis, and progression of chronic hepatitis C: a retrospective study on a large cohort of patients in the United States. J Hepatol. 2004; 40:147-154. [PubMed: 14672626]

7. Walls HL, Magliano DJ, Stevenson CE, et al. Projected progression of the prevalence of obesity in Australia. Obesity. 2012; 20:872-878. [PubMed: 21233805]

8. Wang Y, Beydoun MA. The obesity epidemic in the United States—gender, age, socioeconomic, racial/ethnic, and geographic characteristics: a systematic review and meta-regression analysis. Epidemiol Rev. 2007; 29:6-28. [PubMed: 17510091]

9. Thein HH, Walter SR, Gidding HF, et al. Trends in incidence of hepatocellular carcinoma after diagnosis of hepatitis B or C infection: a population-based cohort study, 1992-2007. J Viral Hepatitis. 2011; 18:e232-e241.

10. Sievert W, Altraif I, Razavi HA, et al. A systematic review of hepatitis C virus epidemiology in Asia, Australia and Egypt. Liver Int. 2011; 31:61-80. [PubMed: 21651703]

11. Cai T, Dufour JF, Muellhaupt B, et al. Viral genotype-specific role of PNPLA3, PPARG, MTTP, and IL28B in hepatitis C virus-associated steatosis. J Hepatol. 2011; 55:529-535. [PubMed: 21236304]

12. Tillmann HL, Patel K, Muir AJ, et al. Beneficial IL28B genotype associated with lower frequency of hepatic steatosis in patients with chronic hepatitis C. J Hepatol. 2011; 55:1195-1200. [PubMed: 21703198]

13. Romeo S, Kozlitina J, Xing C, et al. Genetic variation in PNPLA3 confers susceptibility to nonalcoholic fatty liver disease. Nat Genet. 2008; 40:1461-1465. [PubMed: 18820647]

14. Sookoian S, Castano GO, Burgueno AL, Gianotti TF, Rosselli MS, Pirola CJ. A nonsynonymous gene variant in the adiponutrin gene is associated with nonalcoholic fatty liver disease severity. $\mathrm{J}$ Lipid Res. 2009; 50:2111-2116. [PubMed: 19738004]

15. Speliotes EK, Butler JL, Palmer CD, Voight BF, Hirschhorn JN. PNPLA3 variants specifically confer increased risk for histologic nonalcoholic fatty liver disease but not metabolic disease. Hepatology. 2010; 52:904-912. [PubMed: 20648472]

16. Valenti L, Rumi M, Galmozzi E, et al. Patatin-Like phospholipase domain-containing 3 I148 M polymorphism, steatosis, and liver damage in chronic hepatitis C. Hepatology. 2011; 53:791-799. [PubMed: 21319195]

17. Trepo E, Pradat P, Potthoff A, et al. Impact of patatin-like phospholipase-3 (rs738409 C > G) polymorphism on fibrosis progression and steatosis in chronic hepatitis C. Hepatology. 2011; 54:60-69. [PubMed: 21488075]

18. McHutchison JG, Lawitz EJ, Shiffman ML, et al. Peginterferon alfa-2b or alfa-2a with ribavirin for treatment of hepatitis C infection. N Engl J Med. 2009; 361:580-593. [PubMed: 19625712]

19. Ge D, Fellay J, Thompson AJ, et al. Genetic variation in IL28B predicts hepatitis C treatmentinduced viral clearance. Nature. 2009; 461:399-401. [PubMed: 19684573]

20. Fellay J, Thompson AJ, Ge D, et al. ITPA gene variants protect against anaemia in patients treated for chronic hepatitis C. Nature. 2010; 464:405-408. [PubMed: 20173735] 
21. Price AL, Patterson NJ, Plenge RM, Weinblatt ME, Shadick NA, Reich D. Principal components analysis corrects for stratification in genome-wide association studies. Nat Genet. 2006; 38:904909. [PubMed: 16862161]

22. Johnson AD, Handsaker RE, Pulit SL, Nizzari MM, O'Donnell CJ, de Bakker PIW. SNAP: a webbased tool for identification and annotation of proxy SNPs using HapMap. Bioinformatics. 2008; 24:2938-2939. [PubMed: 18974171]

23. The 1000 Genomes Project Consortium. A map of human genome variation from population-scale sequencing. Nature. 2010; 467:1061-1073. [PubMed: 20981092]

24. Purcell S, Neale B, Todd-Brown K, et al. PLINK: a tool set for whole-genome association and population-based linkage analyses. Am J Hum Genet. 2007; 81:559-575. [PubMed: 17701901]

25. Hickman IJ, Clouston AD, Macdonald GA, et al. Effect of weight reduction on liver histology and biochemistry in patients with chronic hepatitis C. Gut. 2002; 51:89-94. [PubMed: 12077098]

26. Appel LJ, Clark JM, Yeh H-C, et al. Comparative effectiveness of weight-loss interventions in clinical practice. N Engl J Med. 2011; 365:1959-1968. [PubMed: 22085317]

27. Monto A, Patel K, Bostrom A, et al. Risks of a range of alcohol intake on hepatitis C-related fibrosis. Hepatology. 2004; 39:826-834. [PubMed: 14999703]

28. Stickel F, Buch S, Lau K, et al. Genetic variation in the PNPLA3 gene is associated with alcoholic liver injury in Caucasians. Hepatology. 2011; 53:86-95. [PubMed: 21254164]

29. Trepo E, Gustot T, Degré D, et al. Common polymorphism in the PNPLA3/adiponutrin gene confers higher risk of cirrhosis and liver damage in alcoholic liver disease. J Hepatol. 2011; 55:906-912. [PubMed: 21334404]

30. Stickel F, Trepo E, Moreno C, Hampe J. Modulation of the effect of PNPLA3 I148M mutation on steatosis and liver damage by alcohol intake in patients with chronic hepatitis C. J Hepatol. 2011; 55:1471-1472.

31. Valenti L, Colombo M, Fargion S. Modulation of the effect of PNPLA3 I148M mutation on steatosis and liver damage by alcohol intake in patients with chronic hepatitis C. J Hepatol. 2011; 55:1470-1471. [PubMed: 22100032]

32. Younossi ZM, Afendy A, Stepanova M, et al. Gene expression profile associated with superimposed non-alcoholic fatty liver disease and hepatic fibrosis in patients with chronic hepatitis C. Liver Int. 2009; 29:1403-1412. [PubMed: 19515216]

33. Honda M, Sakai A, Yamashita T, et al. Hepatic ISG expression is associated with genetic variation in interleukin 28B and the outcome of IFN therapy for chronic hepatitis C. Gastroenterology. 2010; 139:499-509. [PubMed: 20434452]

34. Urban TJ, Thompson AJ, Bradrick SS, et al. IL28B genotype is associated with differential expression of intrahepatic interferon-stimulated genes in patients with chronic hepatitis $\mathrm{C}$. Hepatology. 2010; 52:1888-1896. [PubMed: 20931559]

35. Dill MT, Duong FHT, Vogt JE, et al. Interferon-induced gene expression is a stronger predictor of treatment response than IL28B genotype in patients with hepatitis C. Gastroenterology. 2011; 140:1021e1010-1031e1010. [PubMed: 21111740]

36. Valenti L, Aghemo A, Stattermayer AF. Interaction between IL28B and PNPLA3 genotypes in the pathogenesis of steatosis in chronic hepatitis C non genotype-3 patients. J Hepatol. 2012; 56:1209-1210. [PubMed: 22230871] 


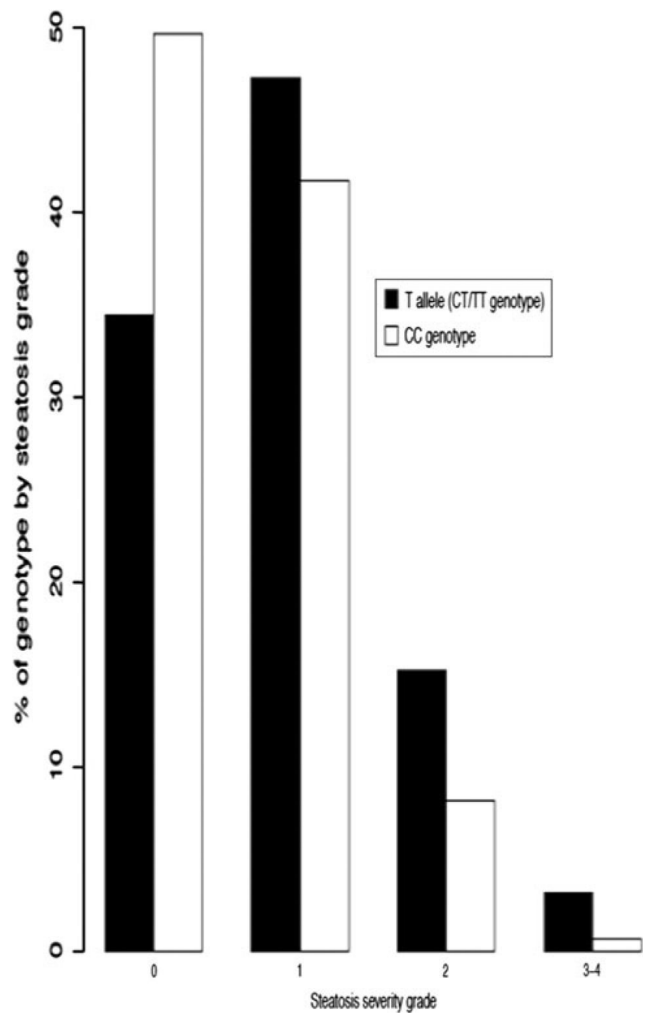

Fig. 1.

Proportion of patients by IL28B genotype (rs12979860, CC vs. CT/TT) by steatosis grade. Patients with the T allele (CT/TT genotypes) have proportionally more severe steatosis than patients with CC genotype (one-sided Wilcoxon rank-sum $p$ value $=4.821 \times 10^{-8}$ ) 


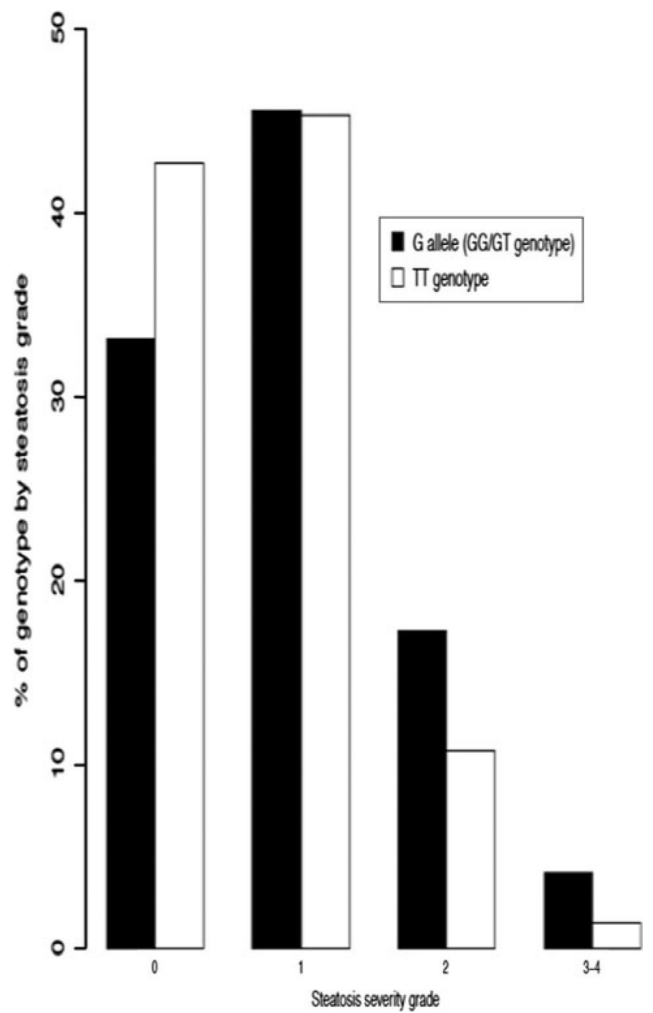

Fig. 2.

Proportion of patients by PNPLA3 genotype (rs2896019, TT vs. GT/GG) by steatosis grade patients with the $\mathrm{G}$ allele (GT/GG genotypes) have proportionally more severe steatosis than patients with TT genotype (one-sided Wilcoxon rank-sum $p$ value $=8.282 \times 10^{-5}$ ) 
Table 1

Clinical characteristics of the study population

\begin{tabular}{|c|c|}
\hline \multicolumn{2}{|l|}{ Cohort characteristic } \\
\hline$n$ & 972 \\
\hline Gender (M/F) & $601 / 371$ \\
\hline Age $( \pm \mathrm{SD})$ & 47.1 ( \pm 7.6 years $)$ \\
\hline BMI $\left(\mathrm{kg} / \mathrm{m}^{2}\right)$ mean, $( \pm \mathrm{SD})$ & $27.8( \pm 4.5)$ \\
\hline $\mathrm{BMI}>30 \mathrm{~kg} / \mathrm{m}^{2} n,(\%)$ & $264(27.2 \%)$ \\
\hline Steatosis grade $0 n,(\%)$ & $387(39.8 \%)$ \\
\hline Steatosis grade $1 n,(\%)$ & $441(45.4 \%)$ \\
\hline Steatosis grade $2 n,(\%)$ & $123(12.7 \%)$ \\
\hline Steatosis grade $3 / 4 n,(\%)$ & $21(2.1 \%)$ \\
\hline Hepatic fibrosis (METAVIR F3-4) $n,(\%)$ & $113(11.6 \%)$ \\
\hline Activity grade (\% with grade $2-3$ ) & $812(83.5 \%)$ \\
\hline Viral load ( $\left.\log _{10} \mathrm{IU}\right)$, median (25-75th percentile) & $6.47(6.03-6.81)$ \\
\hline Fasting glucose (mmol/l), median (25-75th percentile) & $5.10(4.80-5.60)$ \\
\hline Baseline triglycerides (mg/dl), median (25-75th percentile) & $103.6(77.06-148.0)$ \\
\hline Baseline total cholesterol (mg/dl), median (25-75th percentile) & $177.1(155.8-199.2)$ \\
\hline ALT $(\times \mathrm{ULN})$, median $(25-75$ th percentile) & $1.65(1.15-2.63)$ \\
\hline Liver biopsy length (mm) (mean) & 20.9 \\
\hline Number of samples with a biopsy length >15 mm (\%) & $576(59.3 \%)$ \\
\hline $\operatorname{SVR}(\%)$ & $433 / 780(55.5 \%)$ \\
\hline
\end{tabular}

$S D$ standard deviation, $B M I$ body mass index, $I U$ international units, $A L T$ alanine aminotransferase, $U L N$ upper limit of normal, $S V R$ sustained viral response 


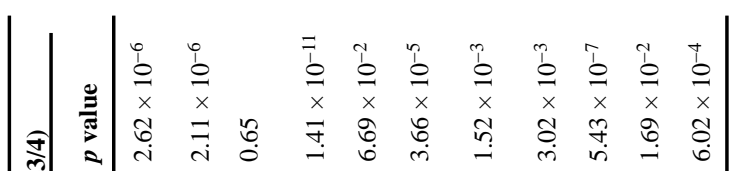

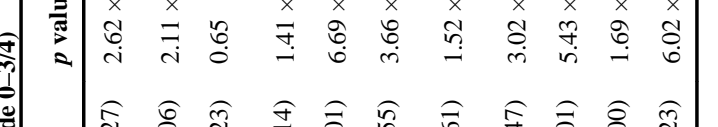

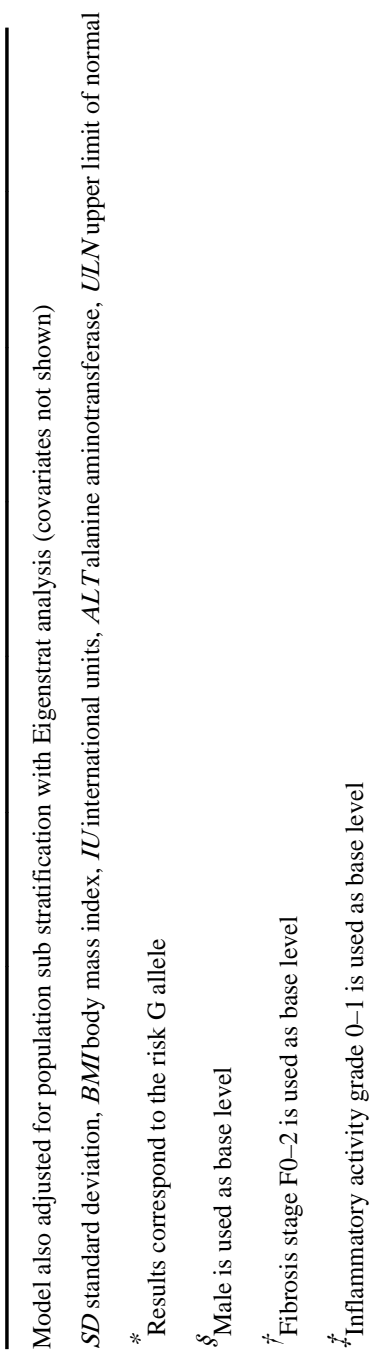

Dig Dis Sci. Author manuscript; available in PMC 2012 December 11. 


\section{Table 4}

Change in hepatic steatosis severity model performance by inclusion of rs12979860 and/or rs2896019 (for model covariates see Tables 2, 3)

\begin{tabular}{lllll}
\hline & No SNP & rs12979860 & rs2896019 & Both \\
\hline${\text { Pseudo } \mathrm{R}^{2}(\%)}^{10.1}$ & 11.7 & 11.2 & 12.7 \\
\hline
\end{tabular}

